shows that only two species of luminous bacteria are present in significant amounts.

The physiology and biochemistry of the more common and well-studied species from marine sources show them to be well adapted to their environment. All will grow in the absence of oxygen and will luminesce at low oxygen concentrations. Many will grow and luminesce at temperatures around $0^{\circ} \mathrm{C}$. Some, however, have relatively complex nutritional requirements in artificial culture, a possible indication that they are not free-living in the seas. As some species tolerate relatively high concentrations of bile salts, it is possible that their natural habitat is the intestines of fish.

Mr. E. S. Trickett (National Institute of Agricultural Engineering, Silsoe) described "Problems of
Radiation Measurement in Relation to Environmental Control". The great difficulties of measuring the distribution of light and heat in glass-houses were pointed out. The wave-length range to be considered reaches from 3,000 to $30,000 \mathrm{~A}$., and well beyond if thermal radiation has to be taken into account. The combination of spatial, temporal, and wave-length factors makes a very formidable problem. When to this is added the need for cheap detectors (existing ones cost several hundred pounds each), because monitoring must be carried out simultaneously at many different points, it is not surprising that research workers are still compelled to conduct growth experiments in glass-houses under very primitive forms of light control.

\title{
DEVELOPMENT OF ARCHITECTURAL SCIENCE
}

\section{A} PUBLICATION* of the University of Sydney Extension Board contains twelve lectures and records of discussions, which were held in the autumn of 1956, on subjects under the general heading of "Structure and Architecture". Section $A$ contains four lectures on mechanics; Section $B$, four lectures on esthetics; and Section $C$, four lectures on construction. The purpose of the lectures is stated to be "to supplement the postgraduate work of architects and architectural assistants and to assist in maintaining interest in the study of the development of structure as related to architecture".

In the first lecture on mechanics, "The Traditional Architectural Forms and the Beginning of the Era of Scientific Structural Design", H. J. Cowan traces the development of the structural form of large buildings through the ages and suggests that its pattern is due to advances in materials and techniques as well as in theory of structures. An interesting account of the main stages in the earlier developments in the latter is included. The next lecture considers "Onedimensional Structures-the Invention of Steel and Reinforced Concrete Construction". It deals with the influence on structural design of the invention of high-strength materials such as steel and reinforced concrete on one hand, and the development of reliable theories for predicting the strength of structural members, on the other. The main result of these advances during the earlier part of the nineteenth century was an increase in the scale of the beam and column type of construction. The third lecture, "Two-dimensional Structures", is mainly concerned with the development of the theory of rigid two-dimensional frames since the middle of the nineteenth century, as a result, initially, of the work of Culmann, Ritter, Maxwell, Mohr and others and, more recently, of the powerful method of moment distribution due to Hardy Cross. The final lecture on mechanics by Prof. Cowan, "The Revival of Curved Roofs and of Three-dimensional Structures", deals with the recent development of integral three-dimensional structures of the shell type, largely as a result of advances in concrete construction techniques. The theory of arch and shell behaviour is outlined, together with the possibility of using

* Architectural Science No. 2: Proceedings of the Symposium on Structure and Architecture. Edited by Prof. Henry J. Cowan. Pp. xii +95-200, (Sydney: Sydney University Extension
Copies obtainable from Academic Press, Pty., Ltd.) 218. experimental (scale model) techniques to circumvent mathematical analysis.

"An Historical Survey of the Influence of Structural Development on Contemporary Architecture" is the title of the first of the lectures on æesthetics. The lecturer, A. N. Baldwinson, suggests that the emotional neutrality of the architecture of many works of the nineteenth century reflects the cessation of the inclusion of the activities of the structural engineer in those of the architect, owing to the increasing complexity of the structural aspect due to new developments. It is noted that nowadays engineers and architects are co-operating, to the advantage of the resthetic aspect. The principal events since 1735 which have influenced architectural development are noted in chronological order in an appendix. The subject of the lecture of F. E. A. Towndrow is "The Theory of Architectural Design in relation to Structures". Some contemporary theories in architectural æesthetics are described and the relevance of æesthetic theories to the work of the engineer is discussed with reference to the opinions of Arup and Nervi. The latter is quoted as saying that æsthetic considerations are fundamental. The place of resthetics in all creative work is outlined, and the training of architects and structural engineers in different ways in separate schools is deplored. The third lecture on rsthetics, "A Painter Looks at Architectural Structure", is by Lloyd Rees and deals with the purely visual aspect. For example, the relationship of buildings to their surroundings is discussed, with emphasis on maintaining local character. It is suggested that one of the most inspiring features of modern structural technique is the covering of large areas without intermediate support. In the final lecture on æsthetics, "The Art of Space", R. B. Grounds asserts that the purpose of architecture is expression, the medium is space and the tool is building. Examples of the concept of space in the architecture of old and new buildings are given and it is suggested that greatness in architecture is only incidentally related to the technological methods employed.

The subject of the first lecture under the general heading of construction is "The Economics of Multistory Buildings". In it, F. Woolard examines the factors which influence the cost of multi-story buildings and separates them into items of capital cost, running cost and maintenance cost, noting that 
these costs are, to some extent, inter-related. Data on costs are given and a plea is made to architects to make cost data available to enable a close study of the factors which influence capital cost to be made. "The Drafting of a Modern Structural Code" is considered in a lecture by R. E. McMillan. It is suggested that such a code should cover standard specifications for materials and fittings and set out design criteria in respect of loads and stresses, but at the same time it should not be restrictive of new materials and design theories. Under the heading "Structural Engineering Practice in the Design of Buildings", R. A. Priddle deals with the function of the structural engineer in relation to the architect and other factors which influence the design of structures for various kinds of building. It is stated that under present building regulations (in Australia, presumably) the reinforced concrete frame is advantageous economically when there are to be more than five stories. Useful data on the cost of different kinds of construction in relation to stanchion spacing are given. The fourth and final lecture on the construction aspect has the title "Structure in Relation to Architectural Practice" and is by C. J. Farrington. The essential qualities of a structure are stated to be : satisfactory performance, economy and beauty, in descending order of importance. It is suggested that beauty has often been sought at the expense of performance and that human activity is hampered by 'fixed structures'. Mr. Farrington concludes: "it may be that some day the building will be nothing more than some kind of aerial field to repel the elements, and allow the human activity to go on unhampered beneath it".

T. M. Charlton

\section{PLASMA APPROACH TO METALLIC CONDUCTION \\ By DR. LOUIS GOLD \\ Massachusetts Institute of Technology, Cambridge, Massachusetts}

\begin{abstract}
RESENT theory of metallic conduction is founded upon the individual electron behaviour as first conceived by Drude and later modified by Sommerfeld to allow for quantum considerations which introduce Fermi-Dirac statistics. It is rather strange that whereas plasma interaction has been invoked to account for the stopping power of metals ${ }^{1}$ for incident high-energy particles and, furthermore, to explain the Ruthemann-Lang experiments ${ }^{2}$ in particular, no theory has been advanced incorporating the plasma contribution to electrical conduction.

It is the purpose of this communication to show that a plasma approach to metallic conduction introduces a type of behaviour that is most suggestive for superconductivity. Indeed, normal and superconductive response may be construed as limiting modes of metallic conduction.

Recent work on plasma oscillations in gases ${ }^{2}$ provides an interesting basis for viewing the conduction process in metals. The model employed is very much like that of the Bardeen and Frohlich quantum mechanical analysis ${ }^{4}$. The metal is taken to be a jelly of positive charge, vibrating as an elastic continuum, in which an electron gas (of charge density equal to that of the ions) is essentially free to move and interact within the matrix jelly. Correlation, exchange and band structure are ignored in this elementary approach. This formulation now embraces both the collective and individual electron interactions.
\end{abstract}

Dynamics of the plasma model for metals. The Boltzmann transport formalism is circumvented by making use of a phenomenological description, as was done in the Drude theory. Thus the individual damped acceleration of an average electron may be expressed as

$$
m \dot{v}+v m \mathbf{v}=q \mathbf{E}
$$

where $v$ embodies collisional damping associated with impurity and lattice scattering.

Collective electron interaction next enters via Poisson's relation, which expresses the long-range coulombic forces acting :

$$
\nabla \cdot \mathbf{E}=\frac{1}{e_{0}} \rho, \rho=\rho_{N}-\rho_{e}
$$

where $\rho$ is the net charge density composed of the electron- and ion-charge densities, $p_{e}$ and $p_{N}$, respectively. Any disturbance of the plasma will give rise to fluctuations in $p_{e}$ which will be damped out with time. The ponderous ions are relatively immobile so that $\rho_{N}$ is effectively constant, with $\left\langle\rho_{e}\right\rangle=f_{N}$ for neutrality.

In one dimension, with electron motion along the $x$-axis, equations (1) and (2) become:

$$
\begin{array}{r}
m \dot{v}+v m v=q E, \quad E \equiv E_{x} \\
\frac{\partial E}{\partial x}=\frac{1}{e_{0}}\left(p_{e}-\rho_{N}\right)
\end{array}
$$

whereby elimination of $E$ to get a velocity relation then proceods via:

$$
\begin{aligned}
m \ddot{v}+m \dot{v}=q \dot{E} & =q\left(\frac{\partial E}{\partial t}+v \frac{\partial E}{\partial x}\right) \\
& =q\left[\frac{\partial E}{\partial t}+\frac{1}{e_{0}} v\left(\rho_{e}-\rho_{N}\right)\right]
\end{aligned}
$$

The conduction current $J=\rho_{e}{ }^{\imath} \gg \frac{\partial E}{\partial t}$ (displacement current), whence :

$$
\ddot{v}+v \dot{v}+\omega^{2} p-\omega^{2} p \frac{J}{\rho_{N}}=0, \omega^{2} p=\frac{q P X}{m e_{0}}
$$

Thus there are three types of solution for $v(t)$.

The auxiliary part of the solution may be

$$
\begin{aligned}
& \text { (A) Oscillatory, } \omega^{2}=4 \omega^{2} p-\nu^{2}>0 \\
& c \exp \left(-\frac{1}{2} \nu t\right) \sin \frac{1}{2} \omega(t-\delta) \\
& \text { (B) Critically damped, } \omega^{2}=0 \\
& \exp \left(-\frac{1}{2} v t\right)\left(c_{1} t+c_{2}\right)
\end{aligned}
$$

